\title{
An Algorithm of Wireless Sensor Network Based on Free Space Optical Communication
}

\author{
https://doi.org/10.3991/ijoe.v13i07.7292 \\ $\mathrm{Na}$ Zhang \\ Xichang College, Xichang, Sichuan, China \\ 277588639 @qq.com
}

\begin{abstract}
The hierarchical distributed network topology combined with traditional wireless routing algorithm is adopted and improved by through introducing energy model of FSO sensor network to study the method of extend network nodes life in special occasion. The OEEMRP protocol (Optical Efficient Energy Multi-Path Routing Protocol) is also proposed. The protocol also can save the node energy and improve the network survival time. In the simulation, we compare and analyze performance difference of death node number and residual energy in LEACH and OEEMRP algorithm. The result shows that improved routing algorithm can make network node more intensive, and in the condition of large network size, the performance will be improved significantly. In a word, it has significant role in network communication.
\end{abstract}

Keywords-Free Space Optical, wireless sensor, network topology

\section{Introduction}

Communication technology has been developing toward the direction of increasing communication distance and system capacity. The 21 st century is the information era, with the rapid development of mobile broadband and Internet, the demand for mobile business users is increasing. In the future, multi-service bearer will become the important feature of next generation mobile network. Certain wireless network has relatively narrow bandwidth, and seriously interfered by electromagnetic wave, thereby the support of large-capacity data business is restrictive. Free-Space Optical Communication (FSO) is the two-way communication technology, it uses laser beam as carrier to precede information interaction, including the audio and video information, and relies on the forms of point-to-point, point-to-multipoint, mulpoint-to-multipoint in atmosphere. Meanwhile, it can interconnect with the traditional wired, wireless and optical fiber network, and form reliable, high data transmission rate network architecture. These networks also can be applied in the high-speed information system, which integrates cloud computing and data processing about vessels, aircraft, and satellites and land-based.

This paper mainly researches the method of extend network nodes life in special occasion, analyzes the influence of FSO unidirectional links on system, introduces omni-directional smart antenna on the basis of neighbor discovery algorithm and base 
station discovery algorithm. On the premise of physical link established, we adopt the hierarchical distributed control network, propose efficient FSO sensor network routing protocol OEEMRP (Optical Efficient Energy Multi-path Routing Protocol). By introducing multi-path strategy to reduce energy consumption and extend survival time of the network. This paper introduces the FSO network topology structure, constructs sensor network model, analyzes omni-directional smart antennas, adopts hierarchical distributed control network to construct network model. We also emphatically introduce OEEMRP protocol, which includes modeling, neighbor discovery node, the formation of clusters, data transmission, and adopt the tactics of multi-hop strategy to save node energy. By the wireless sensor network of free space optical communication algorithm simulation and analysis, we simulate several scenarios, and analyze the performance difference of OEEMRP and LEACH algorithm, as long as conclude the simulation results on the basis of algorithm.

\section{Methods}

\subsection{FSO network topology}

In general, FSO network can be divided into point-to-point, point-to-multipoint and mesh network structure, and user can choose appropriate topology according to actual application. The point-to-point is suitable for access network, and the link is independent, so its reliability cannot be guaranteed and is the hotspot of current research. Point-to-multipoint, namely, star type network structure, it focus the business to the center node, then the center node access to the Internet. Other node needs to be done through the center node routing forwarding. This kind of network structure is equal to the center control structure, center node is relatively complex, and it has strong routing information processing ability. These routing modes are responsible and the flow control efficiency is higher. If the center node failure, other links will be severely affected. In the sake of net structure, it's mainly advantage is that it has multiple path between nodes communications. Meanwhile, it can complete network load balance and improve the efficiency of routing to some extent. The internal of structure can be divided into fully distributed and hierarchical and distributed control. The former in the aspects of routing configuration, flow distribution and management is equal. The latter divide network into clusters, every cluster has one cluster node and several common nodes inside the cluster, through the cluster heads to forwarding business.

This research considers several application occasions as follow. In the military application, network node is of great significance to the command post for battlefield environment. The failure of certain node may lead to the sharp decline in network connectivity. In the aspects of civil use, in the situation of fire balloon or sparsely populated, which do not have enough power, we should not only consider the communication quality of each nodes, but also consider the node energy consumption problem in the process of communication. Therefore, we propose specific scenario analysis model and the routing algorithm design to tackle the problem on the basis of FSO sensor network. 


\subsection{FSO omni-directional smart antenna}

In the building process of FSO sensor network, node design is a relatively significant part. The atmospheric effect and node relative motion will affect the building of network node link to some extent. Traditional APT technology can meet the demand of relatively static FSO system. However, it is inapplicable for network topology variable structure FSO network. In this paper, we use the FSO sensor network node, which has greater node coverage on the reference of omni-directional smart antenna model. This smart antenna model adopt the design of the cylinder, embed the optical transceiver array unit in the surface and side of cylinder, integrate the control system, laser transmitter with information processing system inside the antenna. Meanwhile, the base of antenna is a rotating platform and a relevant motor.

If the divergence angle of transmission unit is $\theta$ ( $\mathrm{rad}$ ), transmission unit quantity of every row is $\mathrm{N}$. In order to reach the demand of omni-directional antenna, $N \theta=2 \pi$. In practice, the size of $\mathrm{N}$ is limited, and $\theta$ cannot be too large, in case of the receive function of receiving set be influenced. For the sake of omni-

directional, the transmission unit quantity should be adequate, so make $\mathrm{d} 1=2 r_{1}$, there will be:

$$
N=\frac{\pi R}{r_{1}}
$$

In this condition, transmit unit is continuous, but the beam divergence angle is small, so the blind area may be appear. Although the design of smart antenna cannot cover 360 ranges for one hundred percent, it can improve connectivity of communication link on the basis of optimization design of antenna array element distribution and beam control method. Meanwhile, it can be applied in FSO networking.

\subsection{Hierarchical clustering algorithm for wireless sensor networks based on free space communication}

This research adopts effective hierarchical cluster protocol, prolongs survival time of the whole network, further studies routing policy, and considers the energy consumption of the network nodes. Low-energy adaptive clustering hierarchy (LEACH) is the first wireless sensor network low energy consumption of hierarchical cluster algorithm. LEACH applied for continuously monitoring network node and data processing through the central node. The basic idea of this algorithm is that the nodes in the network randomly select the cluster heads according to certain rules in each cycle, in the next cycle the cluster heads will be replaced to ensure energy load equably allocate to each sensor node. Meanwhile, it can reduce single node energy consumption, to prolong the survival of the whole network. In the running process, cluster heads was constantly chosen to build the corresponding cluster of each cluster head in circulation way. Each loop corresponds to a round, and it has two steps including the establishment of clusters and the data transmission. The establishment of clusters can 
be divided into four parts, choose cluster head, generation of broadcast mechanism, formation of the cluster, formation of scheduling mechanism.

In the phase of cluster head selection, sensor node will randomly generate a number $(0 \sim 1)$, then we compare it with threshold value $\mathrm{T}(\mathrm{n})$, and the calculation formula of $\mathrm{T}(\mathrm{n})$ as shown in (2). If the random number is smaller than the threshold value, then the corresponding node should be chosen as cluster head.

$$
T(n)=\left\{\begin{array}{l}
\frac{p}{1-p \times\left(\text { r mod } \frac{1}{p}\right)} \\
0 \quad \text { ifn } \notin G
\end{array} \quad \text { ifn } \in G\right.
$$

In this formula, $\mathrm{p}$ is the percentage of cluster head nodes to all nodes in network, $\mathrm{r}$ is currently executing round number, $\mathrm{G}$ is a cluster node set, which belong to nearly round $1 / \mathrm{p}$ and not been selected for the cluster head. When the cluster head nodes is determined, it wills send broadcast messages to the surrounding neighbor node, in order to alert the other nodes themselves has become a cluster head. Ordinary nodes according to the strength of the received signal to decide which cluster could be joined. In the meantime, the signals will feedback to the corresponding cluster head to finish this process. Then, cluster nodes adopt TDMA method to assign time slot within cluster nodes, transmit their data[1].

In data transmission phase, sensor nodes send data to the cluster head nodes. Received data will be fused in cluster nodes, and then sent to the sink node. Sink node response to the cluster head node information and then send to managed node to finish further processing. Data transmission phase continues for a period of time, start a new process of clusters, and that cycle repeats.

LEACH protocol has two kinds of measures in the aspect of network energy consumption. Firstly, randomly choose "cluster node", cluster head has been adjusted continuously to balancing load. Secondly, cluster head nodes reduce the additional loss about information interaction based on data fusion technology. So, the nodes cooperatively execute work to reduce the probability of death too rapidly.

However, the main drawback of the LEACH routing protocol is that all nodes can communicate with each other, and all nodes can reach the base station. This assumption is only suitable for the small network, and it cannot establish a connection for the larger network.

LEACH protocol routing model is based on single-hop form, when the location of cluster head node is far than base station, these cluster head nodes in the communication process will generate an abundant of energy loss, resulting in cluster head node survival time will be reduced. According to the FSO network model proposed in this paper, in order to reduce the load of the cluster head node, we adopt the hierarchical routing protocol OEEMRP, introduces the "multi-hop" mechanism and the energy model for wireless optical network nodes combined with LEACH mechanism. The aim of this protocol is to provide efficient, energy-saving, reliable communication for FSO sensor networks[2]. OEEMRP protocol is similar to LEACH, it describes cluster 
reconstruction process by the concept of round in the process of running, and each round can be divided into clusters establishment clusters and data transmission.

\section{$3 \quad$ FSO sensor network modeling}

\subsection{Energy model}

Firstly, we introduce the network node energy model. The building of this model refer to the first radio energy model, energy consumption is related to communication distance, as shown in formula (3). When distance d is relatively small, the energy meet free space energy consumption mode, and $n=2$. If $d$ is greater than certain limited values, it meet multipath fading energy consumption mode, and $n=4$.

$$
E=\mathrm{kd}^{n}
$$

FSO sensor node total energy $\left(E_{T X}\right)$ of send data, including optoelectronic device consumed energy $\left(E_{T X}{ }_{P E}\right)$ like coding, modulation, filtering and propagation of signals, also including omni-directional smart antenna structure data transmission energy consumption $\left(E_{P X}\right)$. As shown in (4), $E_{P X}$ is depended on some factors such as distance node send-receive and permissible error rate.

$$
E_{T X}(m, d, \varphi)=E_{T X P E}(m)+E_{p X}(m, d, \varphi)
$$

$E_{P X}$ is proportional to laser scanning area and energy coefficient of specified signal-to-noise ratio. If the size of sending data is $\mathrm{m}$, laser scanning angle is $\varphi$, transmission distance is d, then $E_{P X}$ :

$$
E_{p X}(m, d, \varphi)=m \varepsilon \varphi d^{2}
$$

$\varepsilon$ is free space energy coefficient, namely, the energy consumption of sending each bit data. From (4) (5), we can obtain:

$$
E_{T_{X}}(m, d, \varphi)=m E_{T X}{ }_{-P E}+m \varepsilon \varphi d^{2}
$$

For receiving set, assuming that received each bit energy consumption is $E_{R}$, then total consumption of $\mathrm{m}$ bits is:

$$
E_{R X}=m E_{R}
$$




\subsection{Establishment phase}

After the initialization node is completed, each node uses the neighbor discovery algorithm to find its neighbor nodes, and each node maintains a neighbor routing table. The sensor node randomly generates a random number $(0 \sim 1)$, and if this number is less than $T$ (n) in (2), then the node is selected as cluster head. The cluster head node sends a broadcast message to its neighbor node based on routing table, which contains the cluster head ID, the position of the cluster head $P_{i}\left(X_{i}, Y_{j}\right)$, other cluster head nodes set $\mathrm{CH}_{i}$, the distance $\mathrm{CHD}_{i}$ from other cluster heads and this cluster. The distance between the head and the base station is $\mathrm{CHB}_{i}$. When the cluster head node receives the broadcast messages from other cluster head nodes, it will adds these cluster heads into corresponding to the message set $\mathrm{CH}_{i}$, and calculates the distance and adds them to the set $\mathrm{CHD}_{i}$. When the common node receives the broadcast message of the cluster head node, it will check whether there is a minimum hop of the cluster head in the routing table or not. If not, it adds the broadcast message as the shortest path parameter. Otherwise it sets it as the alternative path. Then the value will be stored it in its own routing table and other neighbor nodes will be informed. Then the normal node precedes the cluster establishment by sending a request message to join the cluster head nearest to itself.

\subsection{Data transmission phase}

In former phase the cluster head node is selected, each cluster head node sends the message to the base station, which containing its own information and through the mode of hop or multi-hop. The base station records the cluster head information into its own routing table. The base station selects the nearest cluster head as the next hop on the basis of the value of CHBi. The selected cluster head chooses the shortest next hop cluster head according to the path information stored in its own routing table. So each cluster head node chooses an optimal path to reach the base station. The FSO sensor nodes in each cluster send the data to their corresponding cluster head nodes[3], and the cluster head node aggregates the received data and sends it to the sink node based on optimal path. If corresponding cluster node fails, the alternative path which stored in routing table will send the data. The intra-cluster sensor nodes will precede the communication based on the TDMA time slot assigned by cluster head [4 5]. Moreover, each cluster can avoid collisions with other cluster nodes by combining with Carrier Sense (CSMA) multiple access, CDMA encoding. 


\section{$4 \quad$ Results}

\subsection{Simulation parameter setting}

This chapter refers to above FSO sensor network topological structure and energy model, simulate the OEEMRP and LEACH effect of network working life.

In the simulation, we randomly distribute several nodes through the square area, and the base station node coordinates are alterable. For easier compare two algorithm performance, we set the initial energy is $0.05 \mathrm{~J}$. Energy coefficient $\varepsilon$ relate to optical receive unit receiving sensitivity and error rate [6 8]. If energy coefficient $\varepsilon$ is $4 \mathrm{pJ} / \mathrm{bit} / \mathrm{m}^{2}, \mathrm{E}_{\mathrm{TX} \_\mathrm{PE}}$ followed the optical transmission chipset of Germany Silicon Line GmbH. The energy to transmit 1 bit data is $4 \mathrm{pJ}$, the length of the packet is 4000 bits. Due to the energy consumption is proportional to the laser scanning angle; we adopt the omni-directional smart antenna model. In the process of simulation, $\varphi$ is $2 \pi / 3$. Specific simulation value is shown in Table 1 .

Table 1. Example table

\begin{tabular}{|l|c|}
\hline \multicolumn{1}{|c|}{ Parameter } & Value \\
\hline Initial energy & $0.05 \mathrm{~J}$ \\
\hline Consumed energy of electronic device $\mathrm{E}_{\mathrm{TX}}$ PE & $4 \mathrm{pJ} / \mathrm{bit}$ \\
\hline Packet length & $4000 \mathrm{bit}$ \\
\hline The energy loss of data aggregation & $5 \mathrm{~nJ} / \mathrm{bit}$ \\
\hline Energy coefficient $\varepsilon$ & $4 \mathrm{pJ} / \mathrm{bit} / \mathrm{m}^{2}$ \\
\hline Consumed energy of receiver $\mathrm{E}_{\mathrm{R}}$ & $60 \mathrm{pJ} / \mathrm{bit}$ \\
\hline
\end{tabular}

\subsection{Scene simulation and analysis}

Number of network node is 100 , base station coordinate is $(120,50)$ 


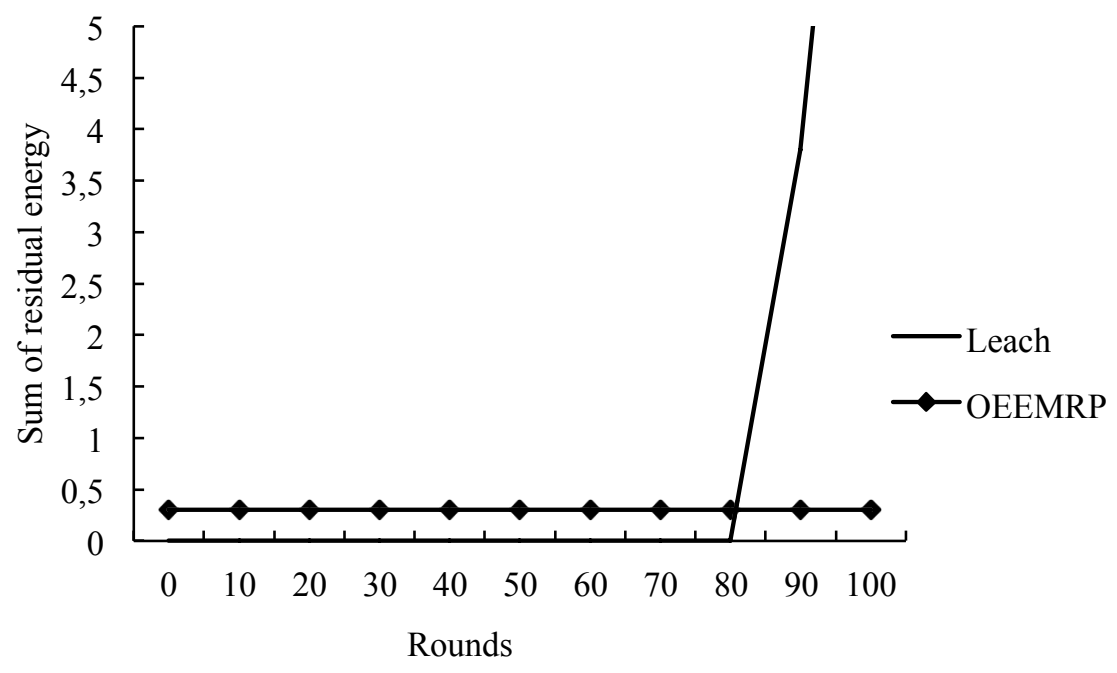

(a) Cluster head proportion $\mathrm{p}$ is 0.05

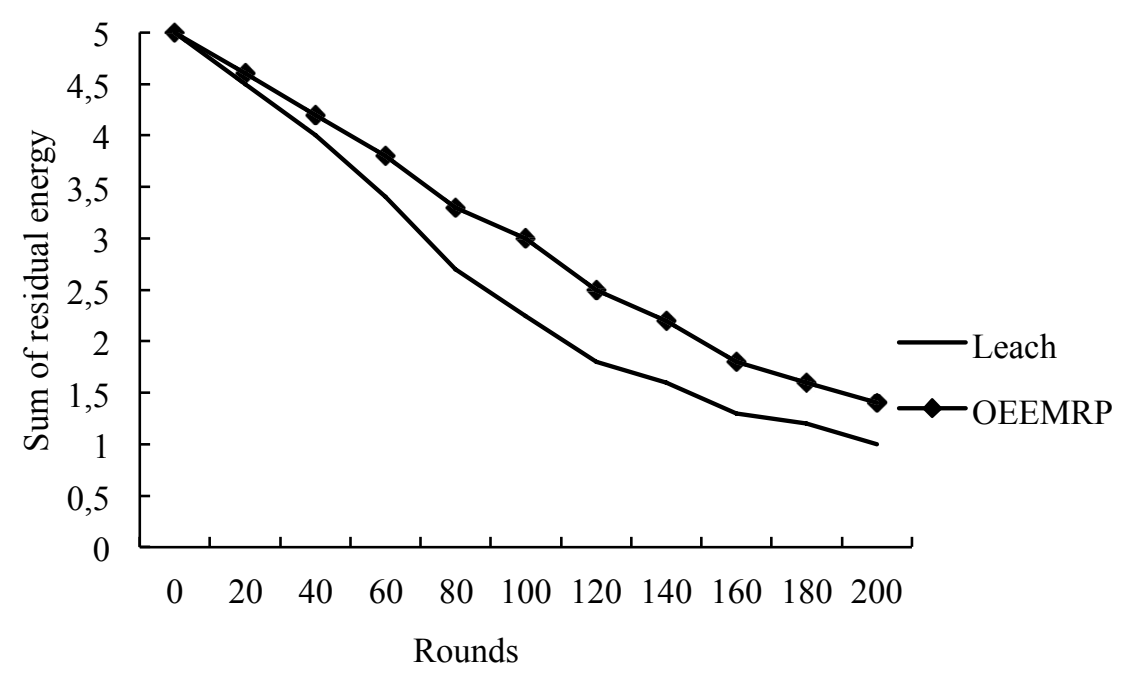

(b) Cluster head proportion $\mathrm{p}$ is 0.1 


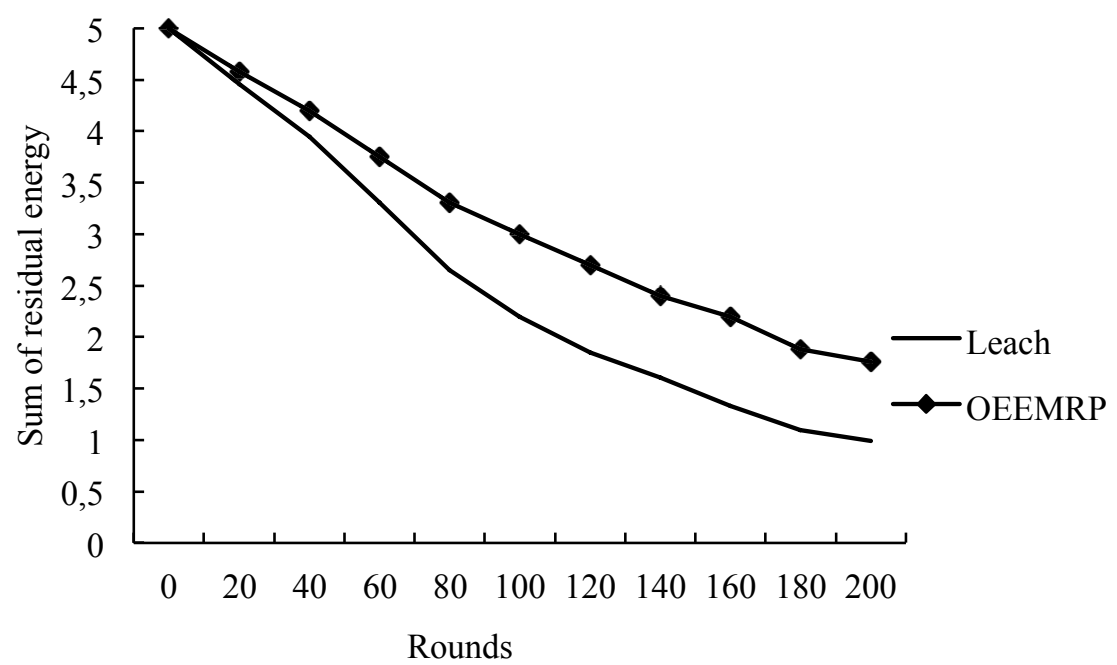

(c) Cluster head proportion $\mathrm{p}$ is 0.2

Fig. 1. Relation curve of different proportion of cluster head node residual energy and the round number.

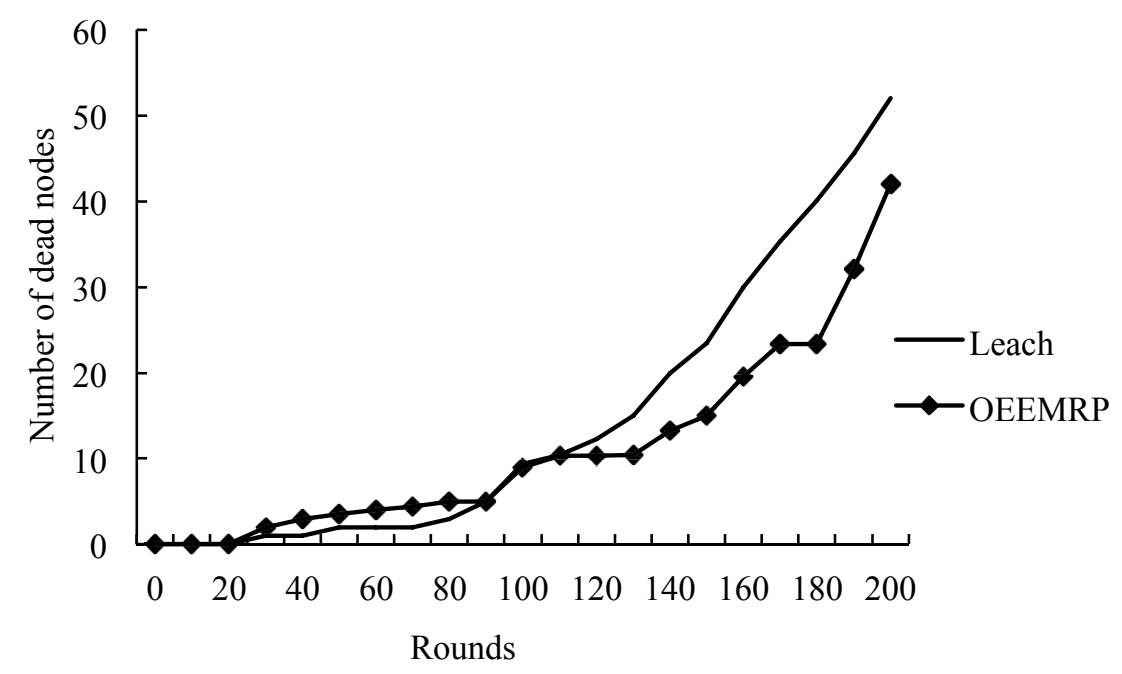

(a) Cluster head proportion $\mathrm{p}$ is 0.05 


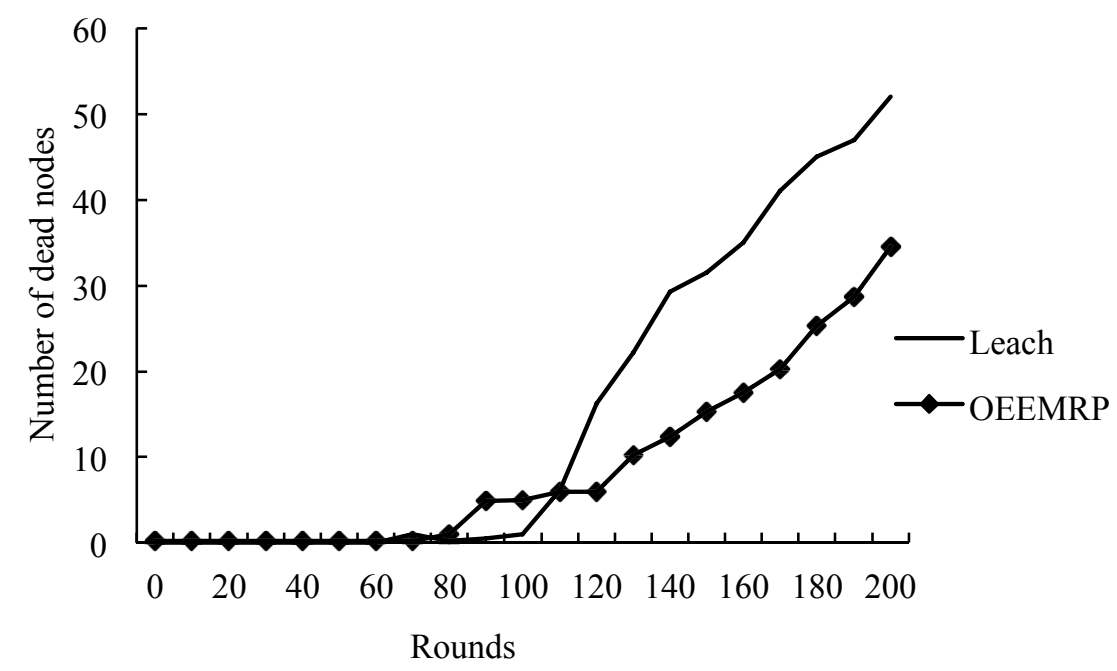

(b) Cluster head proportion $\mathrm{p}$ is 0.1

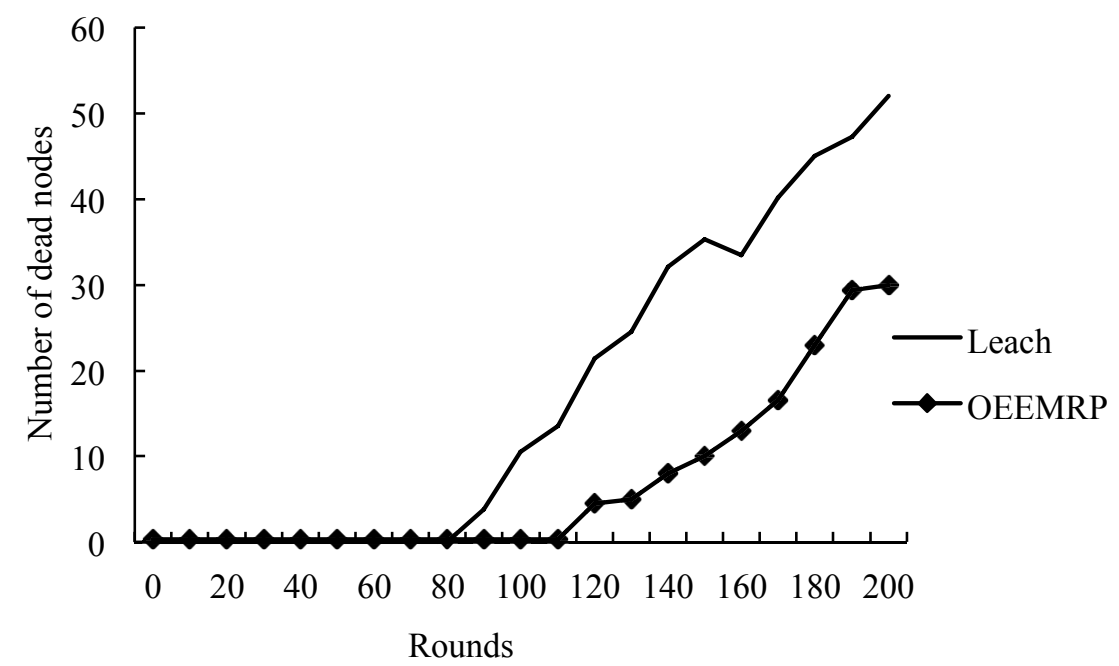

(c) Custer head proportion $\mathrm{p}$ is 0.2

Fig. 2. Relation curve of different proportion of cluster head node number of deaths and the round number.

Figure 1 and figure 2 respectively show the simulation result in the condition of the base station coordinates is $(120,50), 100$ random network nodes. By changing the size of cluster head proportion, we could obtain the node residual energy, node num- 
ber of deaths and round number in the OEEMRP and LEACH algorithm. In Figure 2.a, $\mathrm{p}=0.05$, the round number of producing first death nodes in OEEMRP and LEACH algorithm is 36 and 46. Combined with figure 1, we can find the two kinds of algorithm performance are approximate. In Figure $2 . b, p=0.1$, the round number of producing first death nodes in OEEMRP and LEACH algorithm is57 and 49. In Figure 2.c, $\mathrm{p}=0.2$, the round number of producing first death nodes in OEEMRP and LEACH algorithm is 79 and 63 . It can be seen that when the $p$ value increases, the corresponding number of rounds of first death node in the OEEMRP algorithm increases. And after 200 rounds, energy of nodes and the number of deaths was improved obviously than LEACH algorithm.

In the condition of certain network scale and the base station, results show that if the proportion of cluster head $\mathrm{p}$ is 0.05 , OEEMRP and LEACH algorithm are approximate. With the increase of $\mathrm{p}$, first round death node of OEEMRP is greater than LEACH. After 200 rounds, the remaining nodes energy and the number of nodes in the final survival are greater than LEACH. This is because the smaller the p value will generate smaller cluster heads number in the run time, and the advantage of hierarchy is not obvious. These two algorithms adopt hierarchical routing, when $p$ is 0.05 , the effects are approximate. The greater the value of $p$, the greater the number of cluster nodes. LEACH protocol cluster heads will directly send the information to the base station. OEEMRP uses multi-hop mode to transmit data to the base station on the optimal path. Therefore, the effect of OEEMRP is beneficial than LEACH. Moreover, base station and the network node distance can also affect the performance of the two algorithms. In LEACH algorithms, if the distance of cluster nodes is far than base station, it will cause added energy consumption. In OEEMRP algorithms, we adopt multi-hop strategy, so the performance is improved greatly. The larger the network size, the more advantages OEEMRP algorithm can be reflected.

\section{Conclusion}

Through the simulation analysis, the effectiveness of the algorithm is validated, and the basis for further study of FSO networking is provided. In addition, the multihop strategy is introduced in OEEMRP protocol, and the energy model is changed to reach the demand. But in the process of cluster heads selection, the condition of cluster heads too concentrated or too sparse are not taken into considered. As a result, the two kinds of situation could be generated and the system performance will be influenced. That is to say, although the algorithm has good effectiveness, there are still some problems existing in it that it cannot be widely applied. As a result, the algorithm needs to be optimized further.

\section{References}

[1] Chatzidiamantis, N. D., Michalopoulos, D. S., Kriezis, E. E., et al. (2013). Relay selection protocols for relay-assisted free-space optical systems. Journal of Optical Communications and Networking, 5(1): 92-103. https://doi.org/10.1364/JOCN.5.000092 
[2] Diebold, F. X., \& Y1lmaz, K. (2014). On the network topology of variance decompositions: Measuring the connectedness of financial firms. Journal of Econometrics, 182(1): 119-134. https://doi.org/10.1016/j.jeconom.2014.04.012

[3] Hamedazimi, N., Qazi, Z., Gupta, H., Sekar, V., Das, S. R., Longtin, J. P., ... \& Tanwer, A. (2015). Firefly: A reconfigurable wireless data center fabric using free-space optics. ACM SIGCOMM Computer Communication Review, 44(4): 319-330. https://doi.org/10.1145/2740070.2626328

[4] Kashani, M. A., Safari, M., \& Uysal, M. (2013). Optimal relay placement and diversity analysis of relay-assisted free-space optical communication systems. Journal of Optical Communications and Networking, 5(1): 37-47. https://doi.org/10.1364/JOCN.5.000037

[5] Chan, V. W. (2006). Free-space optical communications. Journal of Lightwave Technology, 24(12): 4750-4762. https://doi.org/10.1109/JLT.2006.885252

[6] Shandilya, S. G., \& Timme, M. (2011). Inferring network topology from complex dynamics. New Journal of Physics, 13(1): 013004. https://doi.org/10.1088/13672630/13/1/013004

[7] Akyildiz, I. F., Su, W., Sankarasubramaniam, Y., \& Cayirci, E. (2002). Wireless sensor networks: a survey. Computer networks, 38(4): 393-422. https://doi.org/10.1016/S13891286(01)00302-4

[8] Zhou, L., Kahn, J. M., \& Pister, K. S. (2003). Corner-cube retroreflectors based on structure-assisted assembly for free-space optical communication. Journal of Microelectromechanical Systems, 12(3): 233-242. https://doi.org/10.1109/JMEMS.2003.809956

\section{Author}

Na Zhang is with Xichang College, Xichang, Sichuan, China (277588639@qq.com).

Article submitted 23 May 2017. Published as resubmitted by the author 27 June 2017. 